\title{
Synthesis and Formation of One-Dimensional Au Nanoparticle Chains*
}

\author{
Ciarán Smyth, ${ }^{\dagger}$ Roseanne Reilly, Yury Rakovich, and Eithne McCabe \\ Semiconductor Photonics Group, School of Physics, Trinity College, Dublin 2, Ireland \\ (Received 6 June 2008; Accepted 16 March 2009; Published 4 April 2009)
}

\begin{abstract}
Structures on the nanoscale have become increasingly prevalent in optoelectronics research in recent years. In particular low dimensional metallic structures have generated interest due to their unique plasmonic properties. We report the synthesis of gold nanoparticles in aqueous solution and the subsequent arrangement of these particles into nanowires-type structures using the dipole-dipole attractive properties of the particles. These nanowire-type structures have differing surface plasmonic properties in comparison to the 0-dimensional nanoparticles due to the 1-dimensional propagation of the plasmon signals. A number of methods were examined for the dipole-dipole interactions to be initiated. Under low $\mathrm{pH}$ conditions $(\sim \mathrm{pH} 3)$ the stabilisers become detached causing a reversible aggregation of the nanoparticles. Through careful tuning of the solution $\mathrm{pH}$ it was examined as to whether only partial removal of the stabilisers was possible with a view to inducing linear aggregation. A second method examined was the removal of the nanoparticles from the aqueous solution and their subsequent dispersal in ethanol. The polar nature of water is seen to interfere with the dipole-dipole interaction once the stabilisers have been removed. Using a less polar solvent was examined, again with regards to initiating nanowire growth.
\end{abstract}

[DOI: 10.1380/ejssnt.2009.327]

Keywords: Gold Nanowires; pH; Surface Plasmon

\section{INTRODUCTION}

The organisation and manipulation of metal nanoparticle assemblies is of a high interest in nanoscience research. The creation of one-dimensional (1D) structures in particular is seen as a particularly useful step as this opens possibilities for bridging structures between nanoscale objects, and the potential for the transmission of surface plasmons across the surface in a given direction. Taking zero-dimensional particles (0D) and processing them towards the desired mass structures is an art form in itself and many techniques exist towards the production of two-dimensional (2D) films of nanoparticles and indeed the $1 \mathrm{D}$ structures mentioned above.

The production of $1 \mathrm{D}$ linear structures of nanoparticles has been studied by using template-based procedures whereby other molecules or structures act as scaffolds to promote the growth of nanowires. Polyelectrolytes, biomolecules and inorganic nanowires have been used to this effect but the subsequent removal of the templates through chemical and physical means can adversely affect the intrinsic properties of the nanowires [1]

Template-free methods for nanowire formation have been less publicised but techniques have been developed recently based on magnetic and electric dipole moments. Methods towards the utilisation of electronic dipole interactions of gold nanoparticles has been reported by Liao et al. in using organic solvents in which to suspend the nanoparticles as this weakens the stabiliser affinity for the particle surface due to the lower polarity of organic solvents such as ethanol [2]. Rakovich et al. report the selfassembly of CdTe nanowires in solution through suspension in a phosphate-buffered solution [3] while Gittins et al. report a decreasing affinity of the stabiliser molecule 4-dimethylaminopyridine (DMAP) for the nanoparticles

* This paper was presented at the 14th International Conference on Solid Films and Surfaces (ICSFS-14), Trinity College Dublin, Ireland, 29 June - 4 July, 2008.

$\dagger$ Corresponding author: ciaran.smyth5@gmail.com

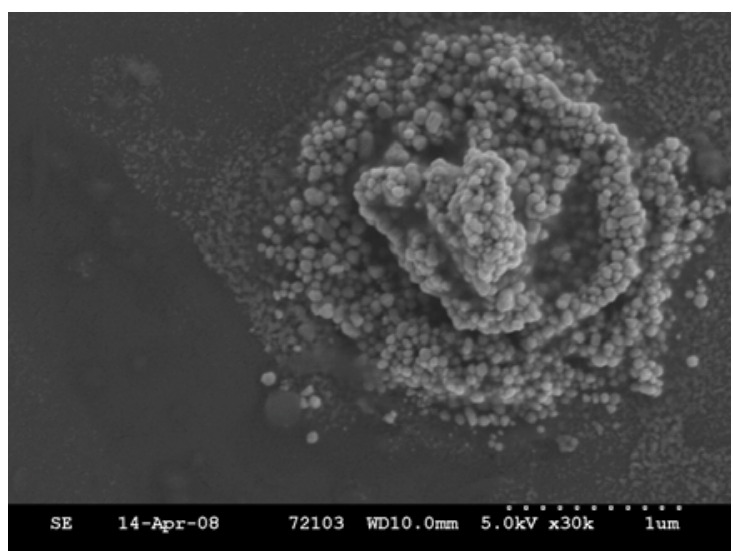

FIG. 1: SEM image taken of a nanoparticle cluster in a dried sample of the aqueous colloidal gold solution. The average nanoparticle diameter was determined to be $65.67 \pm 14.65 \mathrm{~nm}$.

with respect to the $\mathrm{pH}$ of the nanoparticle suspension [4].

Herein it is proposed to examine the changes in the affinity of DMAP for the particle surfaces due to $\mathrm{pH}$ and to examine its potential for inducing dipole-dipole interactions between nanoparticles and any resultant orientation patterns. The nanoparticles were synthesised according to the procedure devised by Gittins et al. and characterisation was carried out using SEM, light microscopy and $\mathrm{UV} / \mathrm{Vis}$ spectroscopy.

\section{EXPERIMENTAL DETAILS}

The gold nanoparticles were synthesised, according to the method described by Gittins et al. [4], in toluene with tetraalkyl ammonium salts acting as a stabiliser molecule to prevent aggregation of the nanoparticles. A phase transfer was then initiated through the addition of an aqueous DMAP solution that acted as the stabiliser once the nanoparticles had become suspended in the aqueous layer. 


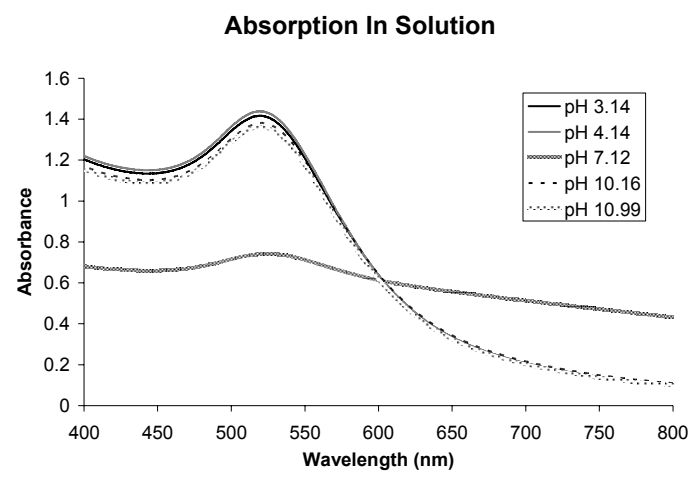

(a)

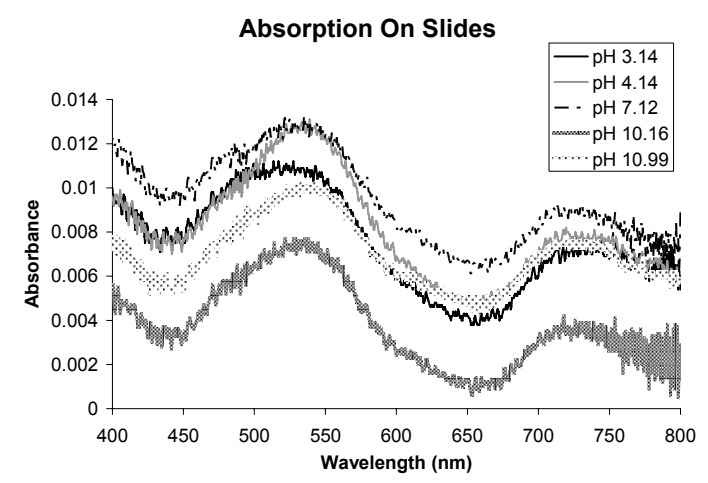

(b)

FIG. 2: Absorption spectra of gold nanoparticle solutions. (a) is of the nanoparticles in aqueous solution while (b) shows the spectra following deposition on a glass microslide.

A series of dilutions were prepared at acidic and basic $\mathrm{pH}$ values using a $\mathrm{pH}$ meter and dilute $\mathrm{HCl}$ and $\mathrm{NaOH}$ solutions to vary the $\mathrm{pH}$ values. The concentrations were varied from $1 / 2,1 / 3,1 / 4$, and $1 / 5$ of the original nanoparticle solution. The acidic $\mathrm{pH}$ values were measured to be 3.14 and 4.14 and those of basic $\mathrm{pH}$ to be 10.16 and 10.99 . A neutral $\mathrm{pH}$ of 7.12 was also used to act as a reference. The solutions were also heated for 30 minutes at $60-70^{\circ} \mathrm{C}$ before being analysed using UV/Vis spectrometry and imaged under a light microscope.

Following the analysis in aqueous solution the respective nanoparticle solutions were centrifuged to remove the bulk of the nanoparticles, before being redispersed in ethanol. This further set of solutions was then again analysed using UV/Vis and light microscopy.

\section{RESULTS AND DISCUSSION}

The result of the nanoparticle synthesis was an $80 \mathrm{~mL}$ solution of the nanoparticles, the concentration of which was derived using the average size of the nanoparticles (measured using SEM-see Fig. 1), the amount of reactant gold atoms $\left(30 \mathrm{mM}\right.$ of $\left.\mathrm{HAuCl}_{4}\right)$ and the face-centred cubic packing structure of gold. The result was a solution of $2.14 \mathrm{nM}$.

This SEM image demonstrates the tendency of the nanoparticles to aggregate once out of solution. The sample shown was of a drop of the originally synthesised aqueous colloidal suspension, allowed to dry at room temperature. By partially disengaging the stabiliser molecules from the surface of the nanoparticles it was envisaged that through dipole-dipole interactions of the nanoparticles a linear aggregation would be initiated. This was attempted through changing of the concentration and the $\mathrm{pH}$ of the solution, and it was hoped to influence the way in which the nanoparticles arranged themselves upon drying.

The solutions were also heated for 30 minutes at 60 $70^{\circ} \mathrm{C}$ as this had previously hinted at the development of a higher wavelength band to signify the longitudinal band of the surface plasmon resonance. On this occasion however, spectra of the nanoparticles showed no such band for any

\section{Absorption On Microslides}

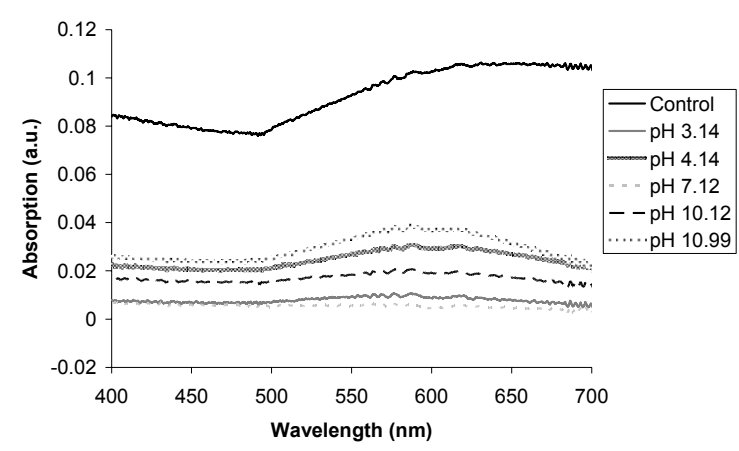

FIG. 3: Absorption spectra of gold nanoparticle solutions dissolved in ethanol.

of the various nanoparticle samples in solution. When deposited and dried onto microslides, however, there is a clear absorption band at around $720 \mathrm{~nm}$, indicating the longitudinal plasmon resonance. These observations are shown in Figs. 2(a) and (b), where the samples displayed are the solutions diluted $1 / 2$ in the respective $\mathrm{pH}$, as this dilution shows the strongest absorbance.

In this procedure $3 \mu \mathrm{L}$ of the solution was dropped onto a microslide and allowed to dry at room temperature before being measured. The solution of $\mathrm{pH} 7.12$ showed a peculiar trend; the colour was markedly different from the acidic and basic solutions, despite the dilution occurring only in de-ionised water. On the microslide the spectrum is more consistent with the rest of the samples, though the presence of the higher wavelength band also suggests little change due to the introduction of acidic and basic pHs.

A second method towards the formation of nanowire arrangements was explored via dispersion of the nanoparticles in ethanol. As a less polar compound than water the affinity for the solvent towards the stabilisers coating the nanoparticles is far reduced. This, in theory, should make the stabilisers less attracted to the nanoparticle surface, with the chance of dipole-dipole interactions between the 


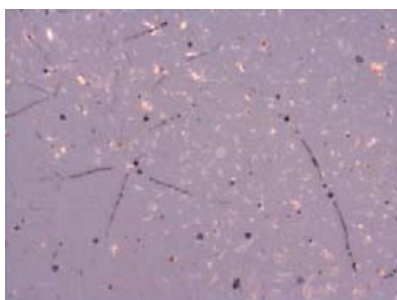

(a)

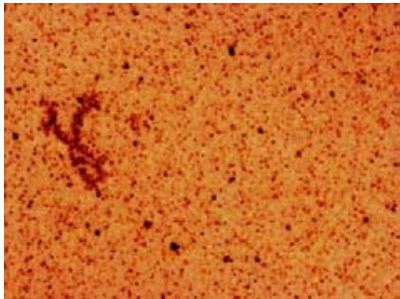

(d)

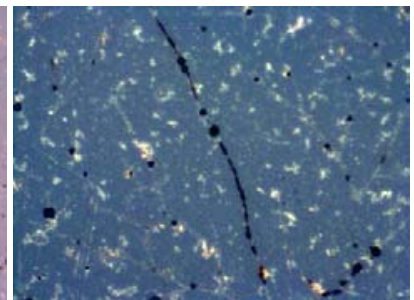

(b)

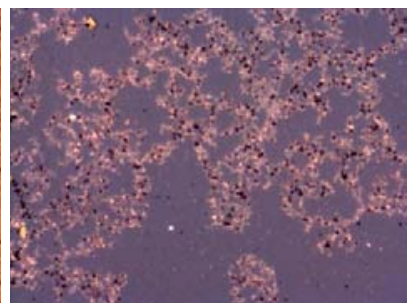

(e)

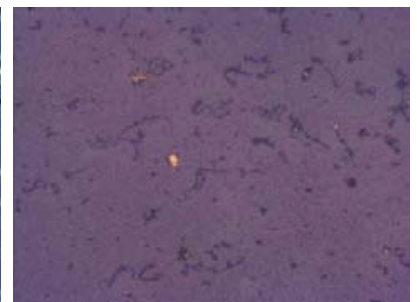

(c)

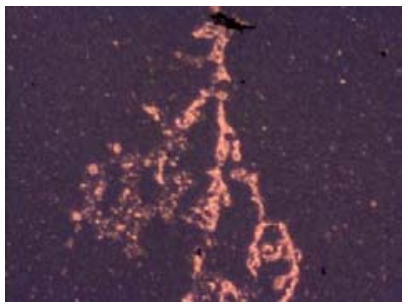

(f)

FIG. 4: (a) A 50× magnification of the $\mathrm{pH} 10.99$ heated sample with a further magnification of $100 \times$ shown in (b). Image (c) shows the pH 10.16 sample at $50 \times$ magnification. (d) is an image of the control sample in ethanol, where a large gold deposit was observed. (e) and (f) show a $50 \times$ image of the $\mathrm{pH} 10.16$ sample in ethanol and a $100 \times$ image of the $\mathrm{pH} 10.99$ sample in ethanol, respectively.

nanoparticles to induce the formation of nanowires.

The solutions in ethanol were prepared according to the experimental details section above and a drop of $3 \mu \mathrm{L}$ was again deposited onto a microslide and the absorption spectrum is shown below in Fig. 3 .

From Fig. 3 it can be seen that there is an extended absorption band when compared to the solutions in aqueous form. The control in the figure refers to the originally synthesised nanoparticle solution, centrifuged and redispersed as with the rest of the samples but with no prior $\mathrm{pH}$ treatment or dilution.

Light microscope images were taken of samples of $3 \mu \mathrm{L}$ volume dried on microslides. Some of the images of the samples are shown below in Fig. 4 and focus on the $\mathrm{pH}$ 10.16 and 10.99 samples at $1 / 2$ dilution, as these posed the greatest suggestion of linear formations.

As can be seen in the images there appear to be some form of linear structures in aqueous solution. Figure 4(b), taken with $100 \times$ magnification shows strong evidence of a linear arrangement in clusters of nanoparticles. This form of structure was also noticed in the $\mathrm{pH} 10.16$ sample, Fig. 4(c), but was not evident in the lower $\mathrm{pH}$ samples. In the ethanol samples the control is shown in Fig. 4(d) where there was a large gold deposit due to the much higher concentration of nanoparticles in solution. On the surface of the gold layer there was no evidence of any linear formations. In the 10.16 and $10.99 \mathrm{pH}$ samples, Figs. 4(e) and 4(f), there was evidence that the gold lay- ers had some form of linear arrangement, but in more of a fractal arrangement than on the scale of nanowires. A possible alternative is that the $\mathrm{pH}$ treatment of the aqueous samples prior to their redispersion in ethanol instigates a tendency to form a networked structure as opposed to a mass film-type deposition.

\section{CONCLUSIONS}

In this work gold nanoparticles were synthesised and imaged using SEM. In order to try to influence a templatefree aggregation of the particles in $1 \mathrm{D}$, the particles were dispersed in higher and lower $\mathrm{pH}$ solutions so as to investigate potential dipole-dipole alignment. At higher $\mathrm{pH}$ 's there was evidence from the UV/Vis spectra and light microscope images to suggest that this might be possible, although more investigation is required. A second method involving the redispersion of the particles in ethanol was also investigated, but while the UV/Vis spectra again suggested higher wavelength absorption bands the microscope images showed more of a fractal-type formation.

In conclusion, continuation of the work might consider the higher $\mathrm{pH}$ solutions to show the most promise for efforts to initiate dipole-dipole nanowire-formation. The aqueous solutions show more potential than when suspended in ethanol, but more analysis needs to be carried out on both procedures with SEM and/or AFM.
[1] Z. Tang and N. A. Kotov, Adv. Mater. 17, 951 (2005).

[2] J. Liao, et al., Colloids and Surfaces A 223, 177 (2003).

[3] Y. P. Rakovich, et al., J. Phys. Chem. C 111, 18927 (2007).
[4] D. I. Gittins and F. Caruso, Angew. Chem. Int. Ed. 40, 3001 (2001). 